\title{
Existence results for singular elliptic equations
}

\author{
J. CHABRowski \\ (Received January 16, 1990, Revised June 15, 1990)
}

The purpose of this paper is to study the existence of positive solutions in $\boldsymbol{R}_{n}$ of the singular elliptic equation

$$
L u=-\sum_{i, j=1}^{n} D_{i}\left(a_{i j}(x) D_{j} u\right)+c(x) u=g(x, u),
$$

where the nonlinearity $g$ is defined on $\boldsymbol{R}_{n} \times(0, \infty)$. Solutions of (1), which are defined on $\boldsymbol{R}_{n}$, are called entire solutions. The precise conditions on $g$, to be formulated later, show that equation (1) is a natural extension of the following equation

$$
-\Delta u=f(x) u^{-\gamma} \text { in } \boldsymbol{R}_{n},
$$

where $\gamma>0$ is a constant. The equation ( $\left.1^{\prime}\right)$ is called in the existing literature the Lane-Emden-Fowler equation and arises in the boundary-layer theory of viscous fluids (see [4], [5], [6], [8] and the references given there). In papers [4] and [8] it is assumed that $f(x)$ depends "almost" radially on $x$ in the sense that

$$
c_{1} p(|x|) \leqq f(x) \leqq c_{2} p(|x|),
$$

where $c_{1}>0$ and $c_{2}>0$ are constants and $p(|x|)$ is a positive function satisfying some integrability condition. The existence results are then obtained using the method of sub and supersolutions. In [5] the existence of positive solutions was obtained by replacing ( $\left.1^{\prime}\right)$ with an equivalent operator equation which can be solved using the Schauder-Tichonov fixed point theorem. In this paper we develop ideas from paper [1], where the existence of weak solutions, in the case $g(x, u)=f(x) u^{-\gamma}, 0<\gamma<\infty$, has been considered. Here we consider more general nonlinearities $g$. Our method in based on approximation arguments. We first solve the Dirichlet problem in a bounded domain with zero boundary data. An entire solution is then obtained as a limit of solutions $u_{m}$ of the Dirichlet problems on $\Omega_{m}$, with $\left\{\Omega_{m}\right\}$ exhausting $\boldsymbol{R}_{n}$. The assumptions $\left(g_{1}\right)$ and $\left(g_{2}\right)$ ensure that solutions of the Dirichlet problem in a bounded domain $\Omega$ belong to $W_{1 \mathrm{loc}}^{1,2}(\Omega) \cap C(\bar{\Omega})$. We also point out that under some additional 
assumption a solution $u$ is in $\stackrel{\circ}{W}^{1,2}(\Omega)$. Throughout this paper we assume that $n \geqq 3$ and we extensively use the Sobolev inequality

$$
\|u\|_{\frac{2 n}{n-2}} \leqq S\|D u\|_{2}
$$

which is true for any $u$ in $\stackrel{\circ}{W}^{1,2}(\Omega)$ and for an arbitrary domain $\Omega \subset \boldsymbol{R}_{n}$ with the constant $S>0$ depending only on $n$. The case $n=2$ can be treated in a similar way, with suitable modifications, due to the fact that in this case the Sobolev inequality remains true with $\|\cdot\| \frac{2 n}{n-2}$ replaced with $\|\cdot\|_{p}, 1<p<\infty$, and with $\|D u\|_{2}$ replaced by $\|u\|_{W^{1,2}}$. However, we do not consider this case here. Some results concerning the case $n=1$ can be found in [6].

Finally, I would like to express my gratitude to Professor A. M. Fink for his interest in this research and bringing my attention to the paper [6]

\section{The Dirichlet problem in a bounded domain.}

We commence by studying the Dirichlet problem

$$
\begin{aligned}
& L u=-\sum_{i, j=1}^{n} D_{i}\left(a_{i j}(x) D_{j} u\right)+c(x) u=g(x, u) \text { in } \Omega, \\
& u(x)=0 \text { on } \partial \Omega,
\end{aligned}
$$

where $\Omega \subset \boldsymbol{R}_{n}$ is a bounded domain with the boundary $\partial \Omega$ satisfying the exterior cone condition.

Throughout this section we make the following assumptions

(A) There exists a constant $\lambda>0$ such that

$$
\lambda|\xi|^{2} \leqq \sum_{i, j=1}^{n} a_{i j}(x) \xi_{i} \xi_{j}
$$

for all $x \in \boldsymbol{R}^{n}$ and $\xi \in \boldsymbol{R}_{n}$. Moreover, we assume that $a_{i j}(i, j=1, \cdots, n)$ and $c$ are in $L^{\infty}(\Omega)$ with $c(x) \geqq 0$ on $\Omega$.

The function $g: \Omega \times(0, \infty) \rightarrow(0, \infty)$ is a Carathéodory function, that is, $g(\cdot, u)$ is a measurable function for each $u \in(0, \infty)$ and $g(x, \cdot)$ is continuous on $(0, \infty)$ for a.e. $x \in \Omega$.

Further, we impose the following two conditions on $g$ :

$\left(g_{1}\right)$ for each $a>0$ there exists $f_{a} \in L^{p}(\Omega)$, with $p>n$, such that

$$
g(x, u) \leqq f_{a}(x) \text { on } \Omega \text { for all } a \leqq u<\infty,
$$

$\left(g_{2}\right)$ the function $g(x, \cdot):(0, \infty) \rightarrow(0, \infty)$ is nonincreasing for a. e. $x$ $\in \Omega$.

THEOREM 1. The Dirichlet problem (2), (3) admits a positive solution $u \in W_{\mathrm{loc}}^{1,2}(\Omega) \cap C(\bar{\Omega})$. 
PROOF : Let $\varepsilon>0$ and consider the Dirichlet problem for the equation $(2 \varepsilon)$

$$
L u=g(x,|u|+\varepsilon) \text { in } \Omega,
$$

with the boundary condition (3). By the Schauder fixed point theorem and the Sobolev-Rellich embedding theorem, the problem $(2 \varepsilon)$, (3) has a unique solution $u_{\varepsilon}$ in $\stackrel{\circ}{W}^{1,2}(\bar{\Omega})$, which by $\left(g_{1}\right)$ belongs to $C(\bar{\Omega})$ (see Theorem 8.30 in [6] $)$. The uniqueness follows from the assumption $\left(g_{2}\right)$. It follows from the mximum principle that $u_{\varepsilon}>0$ on $\Omega$. We now show that $\left\{u_{\varepsilon}\right\}$ is an increasing sequence as $\varepsilon \searrow 0$. Let $0<\varepsilon_{1}<\varepsilon_{2}$. Taking $\left(u_{\varepsilon_{1}}-u_{\varepsilon_{2}}\right)_{+}$ as a test function, we obtain on substitution

$$
\begin{aligned}
& \int_{\Omega}\left[\sum_{i, j=1}^{n} a_{i j}(x) D_{i}\left(u_{\varepsilon_{2}}-u_{\varepsilon_{1}}\right)_{+} D_{j}\left(u_{\varepsilon_{2}}-u_{\varepsilon_{1}}\right)_{+}+c(x)\left(u_{\varepsilon_{2}}-u_{\varepsilon_{1}}\right)_{+}^{2}\right] d x \\
& \quad=\int_{\Omega}\left(g\left(x, u_{\varepsilon_{2}}+\varepsilon_{2}\right)-g\left(x, u_{\varepsilon_{1}}+\varepsilon_{1}\right)\right)\left(u_{\varepsilon_{2}}-u_{\varepsilon_{1}}\right)_{+} d x .
\end{aligned}
$$

Since by the condition $\left(g_{2}\right), g\left(x, u_{\varepsilon_{2}}+\varepsilon_{2}\right)-g\left(x, u_{\varepsilon_{1}}+\varepsilon_{1}\right) \leqq 0$ a. e. on the set $\left\{u_{\varepsilon_{2}}>u_{\varepsilon_{1}}\right\}$, we deduce using the ellipticity condition that

$$
\int_{\Omega}\left|D\left(u_{\varepsilon_{2}}-u_{\varepsilon_{1}}\right)_{+}\right|^{2} d x \leqq 0
$$

and consequently $u_{\varepsilon_{2}} \leqq u_{\varepsilon_{1}}$ a. e. on $\Omega$. In the next step of the proof we show that the sequence $\left\{u_{\varepsilon}+\varepsilon\right\}$ is decreasing as $\varepsilon \backslash 0$. Let $\varepsilon_{1}>\varepsilon_{2}$ and since $u_{\varepsilon_{1}}-u_{\varepsilon_{2}}=0$ on $\partial \Omega,\left(u_{\varepsilon_{1}}+\varepsilon_{1}-u_{\varepsilon_{2}}-\varepsilon_{2}\right)_{-} \in W^{1,2}(\Omega)$ and on substitution we obtain

$$
\begin{gathered}
-\int_{\Omega}\left[\sum_{i, j=1}^{n} a_{i j}(x) D_{i}\left(u_{\varepsilon_{1}}+\varepsilon_{1}-u_{\varepsilon_{2}}-\varepsilon_{2}\right)_{-} D_{j}\left(u_{\varepsilon_{1}}+\varepsilon_{1}-u_{\varepsilon_{2}}-\varepsilon_{2}\right)_{-}\right. \\
\left.+c(x)\left(u_{\varepsilon_{1}}+\varepsilon_{1}-u_{\varepsilon_{2}}-\varepsilon_{2}\right)_{-}^{2}\right] d x \\
=\int_{\Omega}\left(g\left(x, u_{\varepsilon_{1}}+\varepsilon_{1}\right)-g\left(x, u_{\varepsilon_{2}}+\varepsilon_{2}\right)\right)\left(u_{\varepsilon_{1}}+\varepsilon_{1}-u_{\varepsilon_{2}}-\varepsilon_{2}\right)_{-} d x \\
+\int_{\Omega} c(x)\left(\varepsilon_{1}-\varepsilon_{2}\right)\left(u_{\varepsilon_{1}}+\varepsilon_{1}-u_{\varepsilon_{2}}-\varepsilon_{2}\right)_{-} d x .
\end{gathered}
$$

It is easy to see that the right hand side is nonnegative and, as before, we conclude that $\left|D\left(u_{\varepsilon_{1}}+\varepsilon_{1}-u_{\varepsilon_{2}}-\varepsilon_{2}\right)_{-}\right|=0$ a. e. on $\Omega$, that is $u_{\varepsilon_{1}}+\varepsilon_{1} \geqq u_{\varepsilon_{2}}+\varepsilon_{2}$ a. e. on $\Omega$. From these two claims we see that if $0<\varepsilon<\delta$, Then

$$
0<u_{\varepsilon}-u_{\delta}<\delta-\varepsilon \text { on } \bar{\Omega} \text {. }
$$

This means that there exists $u \in C(\bar{\Omega})$ such that $\lim _{\delta \rightarrow 0} u_{\delta}=u$ uniformly on $\bar{\Omega}$. We now show that $u \in W_{10 \mathrm{lc}}^{1,2}(\Omega)$ and satisfies (2) in the distributional sense. Let $B\left(x_{0}, r\right)$ be a ball with a center at $x_{0}$ of radius $r$ and assume 
that $\overline{B\left(x_{0}, 2 r\right)} \subset \Omega$. Let $\Phi$ be a function in $C^{1}\left(\boldsymbol{R}_{n}\right)$ such that $\Phi(x)=1$ on $B\left(x_{0}, r\right), \Phi(x)=0$ on $\boldsymbol{R}_{n}-B\left(x_{0}, 2 r\right)$ and $0<\Phi(x) \leqq 1$ on $\boldsymbol{R}_{n}$. Taking $u_{\varepsilon} \Phi^{2}$ as a test function we obtain on substitution

$$
\begin{aligned}
& \int_{\Omega}\left[\sum_{i j=1}^{n} a_{i j} D_{i} u_{\varepsilon} D_{i} u_{\varepsilon} D_{j} u_{\varepsilon} \Phi^{2}+2 \sum_{i j=1}^{n} a_{i j} D_{j} u_{\varepsilon} u_{\varepsilon} \Phi D_{i} \Phi+c u_{\varepsilon}^{2} \Phi^{2}\right] d x \\
& \quad=\int_{\Omega} g\left(x, u_{\varepsilon}+\varepsilon\right) u_{\varepsilon} \Phi^{2} d x .
\end{aligned}
$$

Since $u_{\varepsilon}$ is positive and increases to $u$ as $\varepsilon \searrow 0$, we may assume that there exist constants $\varepsilon_{0}>0, a>0$ and $A>0$ such that

$$
a \leqq u_{\varepsilon} \leqq A \text { on supp } \Phi,
$$

for all $0<\varepsilon \leqq \varepsilon_{0}$. Using the Young inequality and the assumptions (A) and $\left(g_{1}\right)$ we easily derive from (4) that

$$
\begin{aligned}
\int_{B\left(x_{0}, r\right)}\left|D u_{\varepsilon}\right|^{2} d x & \leqq C\left[\int_{B\left(x_{0}, 2 r\right)} f_{a} u_{\varepsilon} d x+\int_{B\left(x_{0}, 2 r\right)} u_{\varepsilon}^{2} d x\right] \\
& \leqq C \int_{B\left(x_{0}, 2 r\right)}\left(f_{a} A+A^{2}\right) d x,
\end{aligned}
$$

where $C>0$ is a constant independent of $\varepsilon$. The inequality (5) shows that $\left\{u_{\varepsilon}\right\}$ is bounded in $W_{\text {loc }}^{1,2}(\Omega)$. Finally, the Sobolev-Rellich embedding theorem, applied on each compact subset $K$ of $\Omega$, shows that $u$ satisfies (2) in the distributional sense.

REMARK 1. The assumptions $\left(g_{1}\right)$ and $\left(g_{2}\right)$ are satisfied in each following example:

(a) $g(x, u)=f(x) u^{-r}$, with $0<r<\infty$,

(b) $g(x, u)=\frac{f(x)}{\ln (1+u)}$,

(c) $g(x, u)=\frac{f(x)}{\sin \left(\frac{u}{1+u} \frac{\pi}{2}\right)}$,

(d) $g(x, u)=f(x) \exp \frac{1}{u}$,

(e) $g(x, u)=\frac{f(x)}{\left(|x|^{2}+u\right)^{r}}$, with $0<\gamma<\infty$,

where $f>0$ on $\Omega$ and $f \in L^{P}(\Omega)$, with $p>n$.

The functions $g$ from the examples (a)-(d) have the property that $\lim _{u \rightarrow+0} g(x, u)=\infty$ for all $x \in \Omega$. Theorem 1 is related to the results of papers [2-3], where the existence of classical solutions have been investigated under a different set of assumptions including $\lim _{u \rightarrow 0} g(x, u)=\infty$ 
uniformly on $\Omega$.

We now impose an additional assumption on $g$ guaranteeing that $u \in$ $W^{1,2}(\Omega)$.

THEOREM 2. Suppose that there exist constants $b>0$ and $0<\alpha \leqq 1$ and a function $f \in L^{1}(\Omega)$ such that

$$
g(x, u) u^{\alpha} \leqq f(x)
$$

for all $u \in(0, b]$ and a.e. on $\Omega$. Then the solution $u$ of the problem (2), (3) belongs to $W^{1,2}(\Omega) \cap C(\bar{\Omega})$.

PROOF: The proof is straightforward. Let $u_{\varepsilon}$ be a solution of the problem $(2 \varepsilon)$, (3). Taking $u_{\varepsilon}$ as a test function, we get on substitution

$$
\begin{gathered}
\int_{\Omega}\left[\sum_{i, j=1}^{n} a_{i j} D_{i} u_{\varepsilon} D_{j} u_{\varepsilon}+c u_{\varepsilon}^{2}\right] d x=\int_{\Omega} g\left(x, u_{\varepsilon}+\varepsilon\right) u_{\varepsilon} d x \\
\leqq \int_{0<u_{\varepsilon}<b} f u_{\varepsilon}^{1-\alpha} d x+\int_{b<u_{\varepsilon}} f_{b} u_{\varepsilon} d x \\
\leqq \int_{\Omega} f A^{1-\alpha} d x+\int_{\Omega} f_{b} A d x
\end{gathered}
$$

where $A=\sup _{\varepsilon>0, x \in \Omega} u_{\varepsilon}(x)$. This inequality, together with the ellipticity condition (A), yields that $\left\{u_{\varepsilon}\right\}$ is bounded in $W^{1,2}(\Omega)$ and the result follows.

REMARK 2. The condition (6) is obviously satisfied in examples (b), (c) and (a), (e) with $0<r \leqq 1$. If (6) holds with $\alpha>1$, one can easily show that

$$
\int_{\Omega}|D u(x)|^{2} u(x)^{\alpha-1} d x<\infty \text { and } u^{\frac{1+\alpha}{2}} \in \stackrel{\circ}{W}^{1,2}(\Omega)
$$

This result has been obtained in the paper [1].

In the next result we briefly examine the behaviour of $D u$ near the boundary. Let $r(x)=\operatorname{dist}(x, \partial \Omega)$ for $x \in \Omega$.

THEOREM 3. Suppose that $\partial \Omega$ is of class $C^{2}$ and that there exist constants $s>0,0<\alpha \leqq 1, b>0$ and a function $f>0$, with $r^{1+s} f \in L^{1}(\Omega)$ such that

$$
g(x, u) u^{\alpha} \leqq f(x) \text { for } 0<u \leqq b \text { and } a . e . x \in \Omega .
$$

Then the solution $u$ of (2), (3) has the property

$$
\int_{\Omega}|D u(x)|^{2} r(x)^{1+s} d x<\infty \text {. }
$$


PROOF : It follows from the regularity of $\partial \Omega$ that there exists $\delta_{0}$ such that $\partial \Omega_{\delta}$ is of class $C^{2}$ for $\delta \in\left(0, \delta_{0}\right]$, where $\Omega_{\delta}=\{x \in \Omega ; \operatorname{dist}(x, \partial \Omega)>\delta\}$ (see [6] Lemma 14.16, p. 355). We now define a function $\rho \in C^{2}(\Omega)$ such that $\rho(x)=r(x)$ on $\Omega-\Omega_{\delta_{0}}$ and $c_{1} r(x) \leqq \rho(x) \leqq c_{2} r(x)$ on $\bar{\Omega}$ for some constants $c_{1}>0$ and $c_{2}>0$. Let $0<\delta<\delta_{0}$ and set

$$
v(x)= \begin{cases}u(x)(\rho(x)-\delta)^{1+s} \text { for } x \in \Omega_{\delta}, \\ 0 \quad \text { for } x \in \Omega-\Omega_{\delta} .\end{cases}
$$

It is clear that $v \in \stackrel{\circ}{W}^{1,2}(\Omega)$ and taking $v$ as a test function we obtain

$$
\begin{aligned}
& \int_{\Omega_{\delta} i, j=1} \sum_{i j}^{n} a_{i j}(x) D_{i} u D_{j} u(\rho-\delta)^{1+s} d x+(1+s) \int_{\Omega_{\delta} i, j=1} \sum_{i j}^{n} a_{i j}(x) D_{i} u u D_{j} \rho(\rho-\delta)^{s} d x \\
& +\int_{\Omega_{\delta}} c(x) u^{2}(\rho-\delta)^{1+s} d x \leqq A^{1-\alpha} \int_{u<b} f(x)(\rho-\delta)^{1+s} d x \\
& +A \int_{u \geqq b} f_{b}(x)(\rho-\delta)^{1+s} d x,
\end{aligned}
$$

where $A=\max _{\bar{\Omega}} u(x)$. We now observe that by the Young inequality we have

$$
\begin{gathered}
(1+s) \int_{\Omega_{\delta} i, j=1} \sum_{i j}^{n}(x) D_{i} u u D_{j} \rho(\rho-\delta)^{s} d x \leqq \frac{\lambda}{2} \int_{\Omega_{\delta}}|D u(x)|^{2}(\rho-\delta)^{s+1} d x \\
+C \int_{\Omega_{\delta}} u(x)^{2}(\rho-\delta)^{s-1} d x,
\end{gathered}
$$

where $C>0$ is a constant depending on $\left\|a_{i j}\right\|_{L_{\infty}}, s$ and $\lambda$. Hence

$$
\begin{aligned}
\frac{\lambda}{2} \int_{\Omega_{\delta}}|D u(x)|^{2}(\rho-\delta)^{1+s} d x \leqq & A^{1-\alpha} \int_{\Omega} f(x) \rho^{1+s} d x+A \int_{\Omega} f_{b}(x) \rho^{1+s} d x \\
& +C A^{2} \int_{\Omega} \rho^{s-1} d x .
\end{aligned}
$$

Since $\int_{\Omega} \rho^{s-1} d x<\infty$, the result follows from the Lebesgue Monotone Convergence Theorem.

REMAMK 3. The assertion of Theorem 3 can be slightly improved if $a_{i j} \in C^{1}\left(\bar{\Omega}-\Omega_{\delta_{0}}\right)$ and the condition (7) holds with $\mathrm{s}=0$. Then the solution $u$ of the problem (2), (3) has the property

$$
\int_{\Omega}|D u(x)|^{2} r(x) d x<\infty \text {. }
$$


Indeed, using a truncation we may assume that $a_{i j} \in C^{1}(\bar{\Omega})$. Then we proceed as in the proof of Theorem 3. Taking

$$
v(x)= \begin{cases}u(x) & (\rho(x)-\delta) \text { for } x \in \Omega_{\delta} \\ 0 & \text { for } x \in \Omega-\Omega_{\delta}\end{cases}
$$

with $0<\delta<\delta_{0}$, as a test function we arrive at the relation (8) with $\mathrm{s}=0$. The second integral can be handled by integration by parts

$$
\begin{aligned}
& \int_{\Omega_{\delta}} \sum_{i j=1}^{n} a_{i j} D_{j} u u D_{i} \rho d x=\frac{1}{2} \int_{\Omega_{\delta}} \sum_{i j=1}^{n} a_{i j} D_{j}\left(u^{2}\right) D_{i} \rho d x= \\
& +\frac{1}{2} \int_{\partial \Omega_{\delta} i j=1} a_{i j}^{n} a^{2} D_{i} \rho D_{j} \rho|D \rho|^{-1} d S_{x}-\frac{1}{2} \int_{\Omega_{\delta}} u^{2} \sum_{i j=1}^{n} D_{j}\left(a_{i j} D_{i} \rho\right) d x,
\end{aligned}
$$

which shows that this integral is bounded independently of $\delta$.

\section{Entire solutions of (1).}

We now use the results of Section 1 to obtain the existence of positive solutions of (1).

We assume that the hypothesis (A) holds on $\boldsymbol{R}_{n}$ and that the nonlinearity $g$ satisfies the Carathéodory condition and moreover

$\left(g_{1}^{\prime}\right)$ For each $a>0$ there exists a positive function $f_{a} \in L_{\mathrm{loc}}^{p}\left(\boldsymbol{R}_{n}\right) \cap$ $L^{\frac{2 n}{n+2}}\left(\boldsymbol{R}_{n}\right)$, with $p>n$, such that

$$
g(x, u) \leqq f_{a}(x)
$$

for all $u \geqq a$ and a. e. on $\boldsymbol{R}_{n}$.

$\left(g_{2}^{\prime}\right)$ The function $g(x \cdot)$ is nonincreasing on $(0, \infty)$ for a. e. $x \in \boldsymbol{R}_{n}$. We need the follwing lemma.

LEMMA 1. For each number $\delta>0$ the equation

$$
L u=g(x, u+\delta) \text { in } \boldsymbol{R}_{n}
$$

admits a positive solution $v^{\delta} \in W_{10 c}^{1,2}\left(\boldsymbol{R}_{n}\right)$ such that $D v^{\delta} \in L^{2}\left(\boldsymbol{R}_{n}\right)$ and $v^{\delta} \in$ $L^{\frac{2 n}{n-2}}\left(\boldsymbol{R}_{n}\right)$.

PROOF : Let $\left\{\Omega_{m}\right\}, m \geqq 1$, be an increasing sequence of bounded domains with smooth boundaries $\left\{\partial \Omega_{m}\right\}$, such that $\boldsymbol{R}_{n}=\cup_{m \geq 1} \Omega_{m}$. For each $m \geqq 1$, the Dirichlet problem

$$
\begin{aligned}
& L u=g(x,|u|+\delta) \text { in } \Omega_{m}, \\
& u(x)=0 \text { on } \partial \Omega_{m}
\end{aligned}
$$


admits a positive solution $v_{m}^{\delta} \in \stackrel{\circ}{W}^{1,2}\left(\Omega_{m}\right) \cap C\left(\bar{\Omega}_{m}\right)$. This follows by applying the Schauder fixed point theorem. We now extend each function $v_{m}^{\delta}$ by 0 outside $\Omega_{m}$. Since $v_{m}^{\delta} \leqq v_{m+1}^{\delta}$ on $\partial \Omega_{m}$ and $g(x, \cdot)$ is deacreasing it is easy to show that sequence $\left\{v_{m}^{\delta}\right\}$ is increasing as $m>\infty$. Let $\lim _{m \rightarrow \infty} v_{m}^{\delta}(x)=$ $v^{\delta}(x)$ on $\boldsymbol{R}_{n}$. The following estimates show that the function $v^{\delta}$ has all desired properties. Indeed, for each $m$ we have

$$
\begin{aligned}
& \int_{\Omega_{m}}\left[\sum_{i, j=1}^{n} a_{i j} D_{i} v_{m}^{\delta} D_{j} v_{m}^{\delta}+c\left(v_{m}^{\delta}\right)^{2}\right] d x=\int_{\Omega_{m}} g\left(x, v_{m}^{\delta}+\delta\right) v_{m}^{\delta} d x \\
& \leqq\left[\int_{\Omega_{m}} f_{\delta}^{\frac{2 n}{n+2}} d x\right]^{\frac{n+2}{2 n}}\left[\int_{\Omega_{m}}\left|v_{m}^{\delta}\right| \frac{2 n}{n-2} d x\right]^{\frac{n-2}{2 n}}
\end{aligned}
$$

On the other hand by the Sobolev inequality we have

$$
\lambda\left[\int_{\Omega_{m}}\left|v_{m}^{\delta}\right| \frac{2 n}{n-2} d x\right]^{\frac{n-2}{n}} \leqq S \lambda \int_{\Omega_{m}}\left|D v_{m}^{\delta}\right|^{2} d x \leqq S \int_{\Omega_{m} i, j=1} \sum_{i j}^{n} D_{i} v_{m}^{\delta} D_{j} v_{m}^{\delta} d x
$$

where $S>0$ is a constant independent of $m$. These two estimates yield that the integrals

$$
\int_{\Omega_{m}}\left|D v_{m}^{\delta}\right|^{2} d x \text { and } \int_{\Omega_{m}}\left|v_{m}^{\delta}\right| \frac{2 n}{n-2} d x
$$

are bounded independently of $m$ and the result easily follows.

THEOREM 4. The equation (1) admits an entire positive solution $u \in$ $W_{10 \mathrm{c}}^{1,2}\left(\boldsymbol{R}_{n}\right)$ such that

$$
v^{\delta}(x) \leqq u(x) \leqq v^{\delta}(x)+\delta \text { on } \boldsymbol{R}_{n}
$$

for each $\delta>0$, where $v^{\delta}$ is a solution of the equation (9), constructed in the proof of Lemma 1 .

PROOF: As in the proof of Lemma 1, let $\left\{\Omega_{m}\right\}$ be sequence of bounded domains with smooth boundaries such that $\boldsymbol{R}_{n}=\cup_{m \geq 1} \Omega_{m}$. According to Theorem 1 for each $m$ the Dirichlet problem

$$
\begin{aligned}
& L u=g(x, u) \text { in } \Omega_{m}, \\
& u(x)=0 \text { on } \partial \Omega_{m}
\end{aligned}
$$

admits a positive solution $u_{m}$ in $W_{\text {loc }}^{1,2}\left(\Omega_{m}\right) \cap C\left(\bar{\Omega}_{m}\right)$. It follows from the proof of Theorem 1 that for each $m, u_{m}(x)=\lim _{\delta \rightarrow 0} v_{m}^{\delta}(x)$ uniformly on $\bar{\Omega}_{m}$, where $v_{m}^{\delta}$ is a solution of the problem (10), (11). Moreover, we have for $0<\varepsilon<\delta$ 


$$
0 \leqq v_{m}^{\varepsilon}-v_{m}^{\delta} \leqq \delta-\varepsilon \text { in } \Omega_{m} .
$$

Letting $\varepsilon \rightarrow 0$ we get

$$
0 \leqq u_{m}-v_{m}^{\delta} \leqq \delta \text { on } \Omega_{m}
$$

and consequently

$$
v_{m}^{\delta} \leqq u_{m} \leqq v_{m}^{\delta}+\delta \text { on } \Omega_{m} .
$$

Let $B\left(x_{0}, r\right)$ be a ball with center at $x_{0}$ of radius $r$. Let $\Phi$ be a $C^{1}$-function defined as in the proof of Theorem 1. We now choose an integer $q \geqq$ 1 such that $\overline{B\left(x_{0}, 2 r\right)} \subset \Omega_{q}$. Since $\left\{v_{m}^{\delta}\right\}$ is as increasing sequence as $m$ $\nearrow \infty$, we have $v_{q}^{\delta}(x) \leqq u_{m}(x)$ for all $m>q$. Let $0<a=\inf \overline{B\left(x_{0}, 2 r\right)} v_{q}^{\delta}(x)$, then taking $u_{m} \Phi^{2}$ as a test function we get

$$
\begin{gathered}
\int_{\Omega_{m}} \sum_{j=1}^{n} a_{i j} D_{i} u_{m} D_{j} u_{m} \Phi^{2} d x+2 \int_{\Omega_{m} i, j=1} \sum_{i j}^{n} a_{i j} D_{i} u_{m} u_{m} D_{j} \Phi \Phi d x+\int_{\Omega_{m}} c u_{m}^{2} \Phi^{2} d x \\
=\int_{\Omega_{m}} g\left(x, u_{m}\right) u_{m} \Phi^{2} d x \leqq \int_{\Omega_{m}} f_{a} u_{m} \Phi^{2} d x .
\end{gathered}
$$

From this inequality we easily derive the following estimate

$$
\int_{\Omega_{m}}\left|D u_{m}\right|^{2} \Phi^{2} d x \leqq K\left(\int_{\Omega_{m}} u_{m}^{2}|D \Phi|^{2} d x+\int_{\Omega_{m}} f_{a} u_{m} \Phi^{2} d x\right),
$$

for some constant $K>0$ independent of $m$. On the other hand we have for $v_{m}^{\delta}$

$$
\begin{gathered}
\int_{\Omega_{m}}\left[\sum_{i, j=1}^{n} a_{i j} D_{i} v_{m}^{\delta} D_{j} v_{m}^{\delta}+c\left(v_{m}^{\delta}\right)^{2}\right] d x=\int_{\Omega_{m}} g\left(x, v_{m}^{\delta}+\delta\right) v_{m}^{\delta} d x \\
\quad \leqq \int_{\Omega_{m}} f_{\delta} v_{m}^{\delta} d x \leqq\left[\int_{\Omega_{m}} f_{\delta}^{\frac{2 n}{n+2}} d x\right]^{\frac{n+2}{2 n}}\left[\int_{\Omega_{m}}\left|v_{m}^{\delta}\right| \frac{2 n}{n-2} d x\right]^{\frac{n-2}{2 n}}
\end{gathered}
$$

Consequently using the ellipticity and the Sobolev inequality we get

$$
\left[\int_{\Omega_{m}}\left|v_{m}^{\delta}\right| \frac{2 n}{n-2} d x\right]^{\frac{n-2}{2 n}} \leqq C\left[\int_{\Omega_{m}} f_{\delta}^{\frac{2 n}{n+2}} d x\right]^{\frac{n+2}{2 n}}
$$

for some constant $C>0$ independent of $m$. Since $\frac{2 n}{n-2}=2+\frac{4}{n-2}$, we deduce from (15), (16) and (17) that the sequence $\left\{u_{m}\right\}$ is bounded in $W_{10 c}^{1,2}\left(\boldsymbol{R}_{n}\right)$ and we may assure that $\lim _{m \rightarrow \infty} u_{m}=u$ strongly in $L^{2}(K)$, and weakly in $W^{1,2}(K)$ for each bounded domain $K \subset \boldsymbol{R}_{n}$. The inequality (12) is a consequence of Lemma 1 and the inequality (15). It is obvious that $u$ 
is a solution of (1) in the distributional sense.

The following result is an analogue of Theorem 2.

THEOREM 5. Suppose that

$$
g(x, u) u^{\alpha} \leqq f(x) \text { on } \boldsymbol{R}_{n} \times(0, a]
$$

for some constants $a>0$ and $0<\alpha \leqq 1$, where $f(x)>0$ on $\boldsymbol{R}_{n}$ and $f \in$ $L^{\frac{2 n}{n+2+\alpha(n-2)}}\left(\boldsymbol{R}_{n}\right)$. Then there exsits an entire positive solution $u$ of (1) such that $D u \in L^{2}\left(\boldsymbol{R}_{n}\right)$ and $u \in L^{\frac{2 n}{n-2}}\left(\boldsymbol{R}_{n}\right)$.

PROOF : It is sufficient to show that the sequence of solutions $\left\{u_{m}\right\}$ of the Dirichlet problems (13), (14) has the properties: (i ) $\left\{u_{m}\right\}$ is bounded in $L \frac{2 n}{n-2}\left(\boldsymbol{R}_{n}\right)$, (ii) $\left\{D u_{m}\right\}$ is bounded in $L^{2}\left(\boldsymbol{R}_{n}\right)$. By Theorem $2 u_{m} \in$ $\stackrel{\circ}{W}^{1,2}\left(\Omega_{m}\right)$ and we extend functions $u_{m}$ by 0 outside $\Omega_{m}$. Taking $u_{m}$ as a test function we obtain on substitution

$$
\begin{aligned}
& \int_{\Omega_{m}}\left[\sum_{i, j=1}^{n} a_{i j} D_{i} u_{m} D_{j} u_{m}+c u_{m}^{2} d x\right] d x \leqq \int_{\Omega_{m}} g\left(x, u_{m}\right) u_{m} d x \\
& \quad \leqq \int_{u_{m} \leqq a} f u_{m}^{1-\alpha} d x+\int_{u_{m} \geq a} f_{a} u_{m} d x \\
& \leqq \\
& \left.\hline \int_{\Omega_{m}} u_{m}^{\frac{2 n}{n-2}} d x\right]^{\frac{(n-2)(1-\alpha)}{2 n}}\left[\int_{\Omega_{m}} f^{\frac{2 n}{n+2+\alpha(n-2)}} d x\right]^{\frac{n+2+\alpha(n-2)}{2 n}} \\
& \quad+\left[\int_{\Omega_{m}} f_{a}^{\frac{2 n}{n+2}} d x\right]^{\frac{n+2}{2 n}}\left[\int_{\Omega_{m}} u_{m}^{\frac{2 n}{n-2}} d x\right]^{\frac{n-2}{2 n}}
\end{aligned}
$$

On the other hand by the Sobolev inequality we have

$$
S^{-1} \lambda\left[\int_{\Omega_{m}} u_{m}^{\frac{2 n}{n-2}} d x\right]^{\frac{n-2}{n}} \leqq \int_{\Omega_{m}} \sum_{j=1}^{n} a_{i j} D_{i} u_{m} D_{j} u_{m} d x
$$

and the combination of these two estimates gives first the boundedness of $\left\{u_{m}\right\}$ in $L^{\frac{2 n}{n-2}}\left(\boldsymbol{R}_{n}\right)$ and then the boundedness of $\left\{D u_{m}\right\}$ and the result follows.

REMARK 4. If (18) holds with $\alpha>1$, we assume that $f \in L^{1}\left(\boldsymbol{R}_{n}\right)$ and

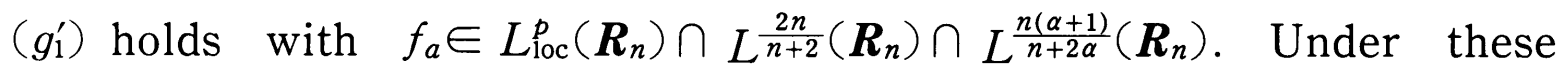
assumptions $\int_{\boldsymbol{R}_{n}} u^{\frac{n(\alpha+1)}{n-2}} d x<\infty$ and $\int_{\boldsymbol{R}_{n}}|D u|^{2} u^{\alpha-1} d x<\infty$. To prove this we use as a test function $u_{m}^{\alpha}$. 


\section{References}

[1] J. CHABROWSKI \& M. KÖNIG, On entire solutions of elliptic equations with singular nonlinearity, Commet. Math. Univ. Carolinae 31, 4 (1990), 645-654.

[2] M. M. COClite \& G. PALMIERI, On a singular nonlinear Dirichlet problem, Comm. in Partial Diff. Equations 14(10) (1989), 1315-1327.

[ 3 ] M. CRANDAll, P. H. RABINOWITZ \& L. TARTAR, On a Dirichlet problem with a singular nonlinearity, Comm. in Partial Diff. Equations 2(2) (1977), 1983-222.

[4] R.DALmasso, Solutions d'équations elliptiques semi-linéaires singuliéres, Ann, Mat. Pura Appl. 4(153) (1989), 191-201.

[5] A. L. EDELSON, Entire solutions of singular elliptic equations, J. Math. Anal. Appl. 139 (2) (1989), 523-532.

[ 6 ] A. M. Fink, J. A. Gatica, G. E. Hernandez \& P. Waltman, Approximation of solutions of singular second order boundary value problems, proprint.

[ 7 ] D. Gilbarg \& N.S. TRUDINGER, "Elliptic partial differential equations of second order," Springer-Verlag, Berlin-Heidelberg-New York-Tokyo (second edition), 1983.

[8] T. KUSANO \& C. A. SWANSON, Entire positive solutions of singular elliptic equations, Japan J. Math. 11(1) (1985), 145-155.

The University of Queensland Department of Mathematics St. Lucia, Queensland Australia, 4067 